\title{
Comparison of Two Pain Assessment Tools, "Facial Expression” and "Critical Care Pain Observation Tool” in Intubated Patients After Cardiac Surgery
}

\author{
Majid Kiavar, ${ }^{1}$ Rasoul Azarfarin, ${ }^{2}$ Ziae Totonchi, ${ }^{2}$ Fatemeh Tavakoli, ${ }^{2, *}$ Azin Alizadehasl, and \\ Mitra Teymouri ${ }^{1}$ \\ ${ }^{1}$ Cardiology Department, Rajaie Cardiovascular Medical and Research Center, Iran University of Medical Sciences, Tehran, Iran \\ ${ }^{2}$ Cardiac Anesthesia Department, Rajaie Cardiovascular Medical and Research Center, Iran University of Medical Sciences, Tehran, Iran \\ ${ }^{*}$ Corresponding author: Fatemeh Tavakoli, Cardiac Anesthesia Department, Rajaie Cardiovascular Medical and Research Center, Iran University of Medical Sciences, Valiasr st. Tehran, \\ Iran. Tel: +98-2123922017, Fax:+98-2122669332, E-mail: razarfarin@yahoo.com; ftm.tavakoli@gmail.com \\ Received 2015 September 26; Revised 2015 October 9; Accepted 2015 October 17
}

\begin{abstract}
Background: Critical-care patients are at higher risk of untreated pain, because they are often unable to communicate owing to altered mental status, tracheal intubation and sedation.

Objectives: This study compared two pain assessment tools on tracheal intubated critically ill patients in a cardiac post-anesthesia care unit, who were unable to communicate verbally. The studied tools were "critical-care pain observation tool (CPOT)" and "facial expression (FE)".

Patients and Methods: This was a prospective study based on diagnostic test evaluation. A sample of 91 intubated patients was selected from cardiac post-anesthesia care unit. Collected data were demographic characteristics, vital signs, FE and CPOT tools' scale. Pain was assessed with CPOT and FE scores five times. The first assessment was performed in at least 3 hours after admission of patients to ICU. Then, the pain intensity was reassessed every 30 minutes. In addition, blood pressure, heart rate, respiratory rate and oxygen saturation were measured simultaneously.

Results: At the first period, the frequency of "severe" pain intensity using the CPOT was 58.2\% and with the FE tool was $67 \%(P=0.001)$. Both tools demonstrated reduction in severity of pain on second and third assessment times. Significantly increasing level of pain and blood pressure due to nursing painful procedures (endo-tracheal suctioning, changing patient's position, etc.), were obtained by CPOT in fourth assessment. FE was not able to detect such important findings $(\kappa=0.249)$. In the fifth step, pain intensity was reduced. The most agreement between the two tools was observed when the reported pain was "severe" ( $\kappa=0.787, \mathrm{P}<0.001)$ and "mild" $(\kappa=0.851, \mathrm{P}<0.001)$.

Conclusions: The sensitivity of CPOT was higher for detection and evaluation of pain in intubated postoperative patients compared with "Facial Expression". Best agreement between these tools was observed in two extremes of pain intensity.
\end{abstract}

Keywords: Pain Assessment, Intensive Care Unit, Cardiac Surgery

\section{Background}

In critical care unit, some factors and patient's condition can alter verbal communication and make pain assessment difficult, including endotracheal intubation, reduced level of consciousness, sedation and administration of paralysing drugs $(1,2)$. After cardiac surgery, tracheal intubated patients in ICU without verbal communication, usually experience painful care after certain treatments (3) since they cannot express their pain verbally, in writing, pointing with fingers or shaking their heads (4). Nursing staff are usually unaware of their pain and it is likely that pain remains untreated (5). Low level of consciousness and being on ventilator or receiving tranquilizers are some samples of this condition (6). Untreated and prolonged pain can affect endocrine, cardiovascular, immune, nervous and musculoskeletal systems and result in chronic pain syn- drome, prolonged hospitalization (7) and increased catecholamine and stress hormones release, which in turn would cause increased blood pressure, increased heart rate and increased oxygen demand and decreased perfusion (8). Heart surgery patients experience extreme pain due to the nature of operation, chest tubes and sternal incision. In fact, stress responses and imbalance between demand and supply for oxygen in myocardium could lead to myocardial ischemia or infarction and may increase mortality rate (9).

Nurses play a vital role in pain assessment (10) and determine pain intensity and perform medical and nonmedical treatment for these patients. Therefore, it is necessary for nurses to be fully aware of a standard and systematic pain evaluation protocol (11). In response to these needs, methods were offered to evaluate pain in 
patients more effectively. Some of such pain assessment tools include facial expression (FE), critical care observation tool (CPOT), non-verbal pain scale (NVPS), faces, legs, activity, cry and consolability scale (FLACC), behavioral pain scale (BPS) and pain assessment in advanced dementia (PAIND) (8).

In 2006, American pain management nursing association recommended using the two tools, BSP and CPOT, for evaluating pain in tracheal intubated and unconscious patients $(8,12)$. As mentioned before, patients who have undergone open heart surgery would experience severe pain (13) and the only regular method in evaluating pain in these patients in studied hospital was facial expression and the only item studied in this method was patient's face. On the other hand, CPOT is used for patients in ICU who have had open heart surgery (14) and is easily performed by caregivers (15).

\section{Objectives}

Therefore, the aim of this study was to evaluate convergence between these two tools for pain assessment and their consistency with hemodynamic changes due to pain in tracheal intubated patients after cardiac surgery.

\section{Patients and Methods}

This was a prospective study. After the research was approved by the institutional ethics committee, evaluating the diagnostic tests was started in a referral university cardiovascular, medical and research center. The study was conducted on patients who had undergone cardiac surgery and met the necessary criteria for the research and were studied until the target sample size $(\mathrm{n}=100)$ was reached. Inclusion criteria were coronary artery bypass grafting surgery, replacing or repairing heart valve by sternotomy and lack of verbal communication due to tracheal intubation, lack of extreme facial damage, movement in at least one body part, patient's age at least 18 and at least 3 hours after any administration of sedatives, analgesics and muscle relaxing agents. Exclusion criteria were opium addiction, receiving above mentioned drugs after surgery during the study, comatose patients, spinal cord damage and body limbs paralysis or weakness and patients with heart failure during the surgery who received high doses of inotropes and vasopressors.

\subsection{Sampling Method and Size}

In this study, the convenience sampling method was used. Patients who had undergone cardiac surgery in a referral university cardiovascular, medical and research center intensive care units, and met the inclusion criteria entered the study. With mathematical relations for KAPPA statistic calculation and general and accidental convergence difference of 0.2 and $\alpha$ type error $=0.5$, and study power of $80 \%$, sample size was calculated as 100 .
Nine people were excluded from study due to incorrect or inadequate data and 91 patients were statistically analyzed.

Data collection tool included demographic information including age, gender, history (diabetes and blood pressure), surgery type and consciousness level, history of drug abuse, sedation and analgesic drugs. Vital signs checklist included systolic and diastolic pressure, pulse and respiratory rate collected by the researcher and recorded by the monitoring device. The behavioral pain scale form for CPOT, which included 4 items of facial movement, body movement, muscle tune and acceptance of ventilator in intubated patients and sound making of non-intubated patients, had two points for each item, 8 points in total. Zero for no pain, and eight for the maximum amount of pain (Table 1). The Behavioral pain scale form for FE including only expressions of patient's face (Figure 1) was completed by the colleague of the project to create blinding.

A score of 1 may be attributed when a change in the patient's facial expression is observed compared with rest assessment (e.g. open eyes, weeping). The drawings were inspired from Prkachin's study (17).

\subsection{Data Collection}

Anesthesia induction was performed by Midazolam 0.1 $\mathrm{mg} / \mathrm{kg}$, Fentanyl 5 - $10 \mu \mathrm{g} / \mathrm{kg}$ or Sufentanil 2.5 - $4 \mu \mathrm{g} / \mathrm{kg}$, Cisatracurium $0.15 \mathrm{mg} / \mathrm{kg}$ and for maintenance of anesthesia we used Midazolam $1 \mu \mathrm{g} / \mathrm{kg} / \mathrm{min}$ and Fentanyl 1 - $3 \mu \mathrm{g} / \mathrm{kg} / \mathrm{h}$ or Sufentanil $0.5-1 \mu \mathrm{g} / \mathrm{kg} / \mathrm{h}$ and Atracurium besilate 0.3 to $0.6 \mathrm{mg} / \mathrm{kg} / \mathrm{h}$. After completing the cardiac surgery, patients were admitted to ICU while intubated. The steps of pain assessment were: 1 ) between 3 - 4 hours after patients were admitted to ICU, the patient gradually emergence from anesthesia and while the patient was still intubated, the researcher would evaluate patient's pain severity using CPOT tool and the research colleague nurse would do the same using facial expression tool, without knowing each other' results (blinded) at Time Zero T0.2). After the initial pain evaluation, the level of analgesia, BP, heart and respiratory rates and the level of oxygen saturation evaluated and recorded every 30 minutes for two hours $\left(T_{1}=30\right.$ minutes, $T_{2}=60 \mathrm{~min}$ utes, $T_{3}=90, T_{4}=120$ minutes $)$. In total, all the units in the research were evaluated 10 times ( 5 times with CPOT and 5 times with FE).

\subsection{Statistical Analysis}

Analysis was performed using SPSS 21.0 for Windows statistical software (SPSS Inc. IBM Corp., Chicago, Illinois, USA). Compatibility of quantitative data distribution with normal distribution was evaluated using one-sample Kolmogorov-Smirnov test. To describe data, mean and standard deviation (SD) for continuous variables and numbers (percentage) for categorical variables were used. To evaluate quantitative variables 
in different time steps, in each group, "repeated measures analysis of variance (ANOVA)" Statistical model was used. Comparison between rating variables (for pain tools) was performed using "Wilcoxon sign-ranked test". To evaluate pain intensity as time passed, Friedman test was used. Convergence between the pain tools was evaluated using weighted kappa with $95 \%$ certainty range. P value equal to or less than $5 \%$ was considered as statistically significant.

\section{Results}

Data was collected from 91 intubated patients in ICU following cardiac surgery. Demographic data of patients are presented (Table 2). Sixty three patients were male $(69.2 \%)$ and the mean age was 56 years. Hypertension and diabetes mellitus were reported as $28.5 \%$ and $24.1 \%$, respectively. Obtained pain evaluation pattern showed severe pain for over a half of patients at To after the effect of anesthetic drugs eliminated; $58.2 \%$ of patients based on CPOT behavioral tool and $67 \%$ based on FE behavioral tool. Then the pain decreased and in minutes 30 and 60, patients experienced almost no extreme pain. In minute 90, a number of patients experienced moderate pain and a smaller number experienced se- vere pain, which intensity decreased in minute 120 . Statistically, the Friedman test was performed on each data from CPOT tool and FE resulted in $\mathrm{P}<0.001$, which showed that changes in pain levels as time passed were significant (Figures 2 and 3 ).

When the patient was conscious, a high level of agreement was observed between the two tools in evaluating pain $(\kappa=0.787)$. However, the level of agreement was weak in $\mathrm{T} 1(\kappa=0.249)$ and $\mathrm{T}_{2}(\kappa=0.555)$, no agreement was observed in $\mathrm{T}_{3}(\kappa=0.166)$, and in $\mathrm{T} 4(\kappa=0.851)$ the agreement was high (Table 3 ). In fact, increase in pain intensity was clearly obvious in minute 90, compared to minutes 60 and 120 using CPOT. Whereas, the FE tool evaluated nearly similar pain severity during these times and showed no increase in the level of pain.

Regarding the correlation of physiologic parameters with level of pain intensity in patients, there was no statistical and clinical significant association between diastolic blood pressure changes and heart rate during the evaluation, and there was only a significant association between systolic blood pressure and the level of pain. Increase in pain intensity with increase of systolic blood pressure was detected and recorded by CPOT tool, whereas this correlation was not observed in FE (Figure 4).

Table 1. Critical Care Observation Tool (CPOT) ${ }^{\mathrm{a}}$

Indicator

-

$\mathbf{0}$

No muscular tension observed: relaxed, neutral

Presence of frown brow

All of the above facial

Facial description

lowering, orbit tightening, and

closed: grimacing

\section{Score} levator contraction: tense

2

Slow cautions movements, touching or rubbing the pain site, seeking attention through movements: protection
Pulling tube, attempting to sit up, moving limbs/thrashing, not following command, striking at staff, trying to climb out of bed: restlessness

\section{Muscle tension (evaluation by passive flexion and extension of upper extremities)} necessarily mean absence of pain): absence of movements

\section{Compliance with the ventilator (intubated patients), OR}

\section{No resistance to passive movements: relaxed}

Resistance to passive movements: tense, rigid
Strong resistance to passive movements, inability to complete them: vary tense or rigid
Vocalization (extubated patients)

\author{
Alarms not activated, easy \\ ventilation: tolerating \\ Ventilator or movement
}

Alarms stop Spontaneously: coughing but tolerating
Asynchrony: blocking ventilation, alarms frequently activated: fighting ventilator

\footnotetext{
$\mathrm{a}_{(16) .}$
}

Talking in normal tone or no sound
Sighing, moaning
Crying out, sobbing 
Kiavar M et al.

Facial expressions

0

Relaxed, neutral (no muscle tension)
$1^{*}$

Tense

(froning. brow lowering. orbit tightening, little levator contraction)

\section{Grimacin}

(contraction of the whole tace: frowning, brow lowering, eyes tightly closed. levator contmcton - mouth may be opened or the patient may be biting the endotracheal tube)

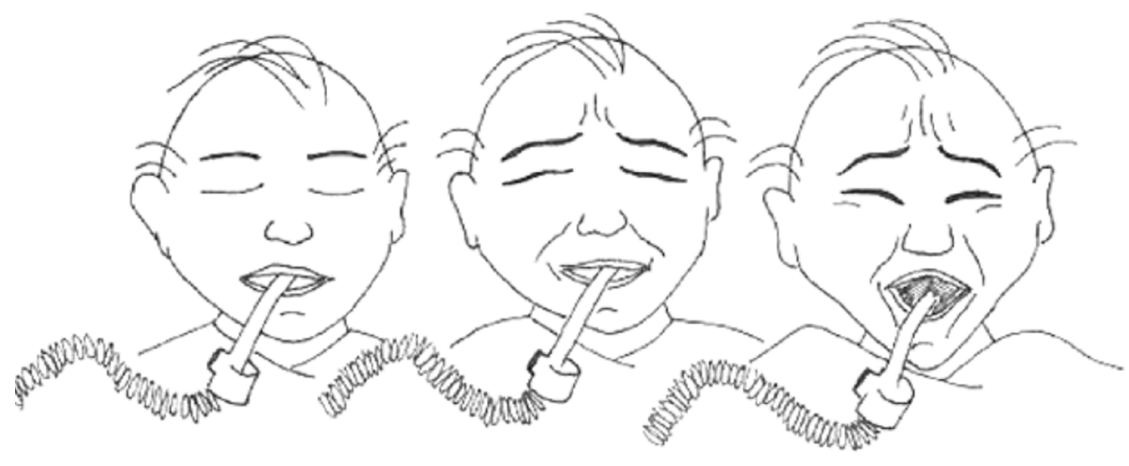

Relaxed. neutral

Tense

Grimacing

0

1

2

Figure 1. Facial Expression Tool for Pain Assessment, Drawings by Caroline Arbour, RN, B.Sc., PhD (student), McGill University

\begin{tabular}{|c|c|}
\hline Variables & Values \\
\hline Age (15 - 83), y & $14.8 \pm 56$ \\
\hline Height (153 - 180), cm & $6.4 \pm 167$ \\
\hline Weight (45 - 105), kg & $12.2 \pm 70$ \\
\hline \multicolumn{2}{|l|}{ Gender } \\
\hline Female & $28(30.76)$ \\
\hline Male & $63(69.24)$ \\
\hline Diabetes mellitus & $22(24.1)$ \\
\hline Hypertension & $26(28.5)$ \\
\hline
\end{tabular}

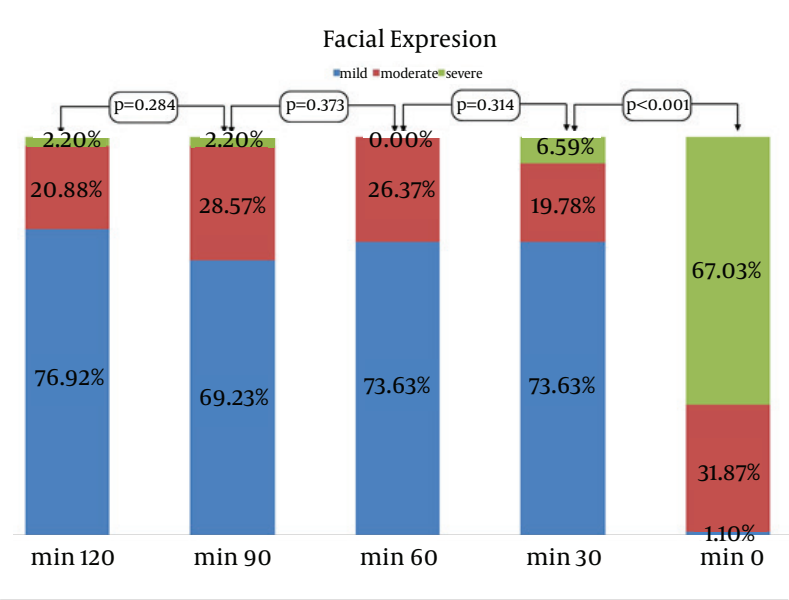

Figure 2. Level of Pain and its Changes in Study Time Intervals Using Facial Expression Tool

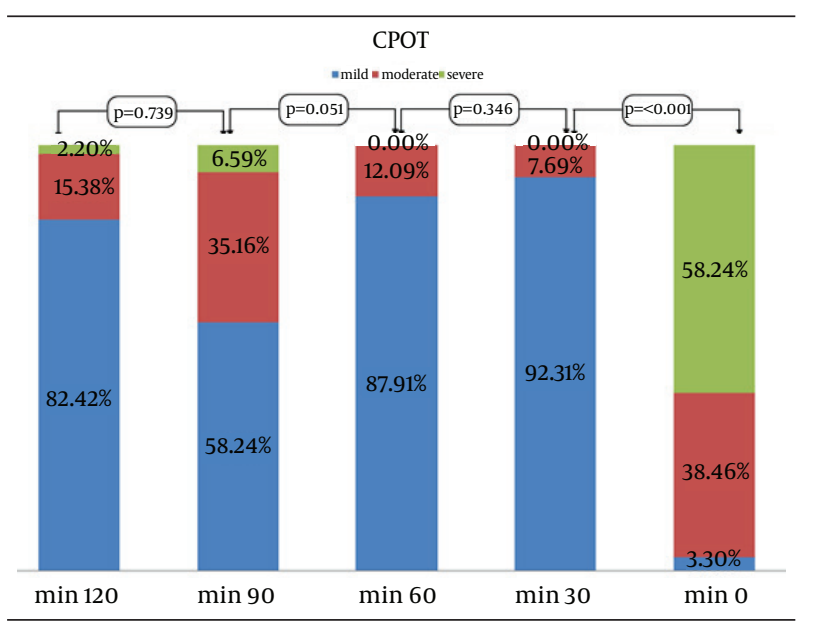

Figure 3. Level of Pain and its Changes in Study Time Intervals Using CPOT Tool 
KiavarM et al.

Table 3. Convergence Between the Two Tools of Behavioural Pain Evaluation CPOT and FE, in Evaluating Patients Pain in Consecutive Evaluation Intervals

\begin{tabular}{lcc}
\hline TIME & Kappa & CI 95\% \\
\hline $\mathbf{0}$ & 0.787 & $(0.675-0.919)$ \\
$\mathbf{3 0}$ & 0.249 & $(0.082-0.477)$ \\
$\mathbf{6 0}$ & 0.555 & $(0.354-0.755)$ \\
$\mathbf{9 0}$ & 0.166 & $(0.0-0.295)$ \\
$\mathbf{1 2 0}$ & 0.851 & $(0.696-0.974)$ \\
\hline
\end{tabular}

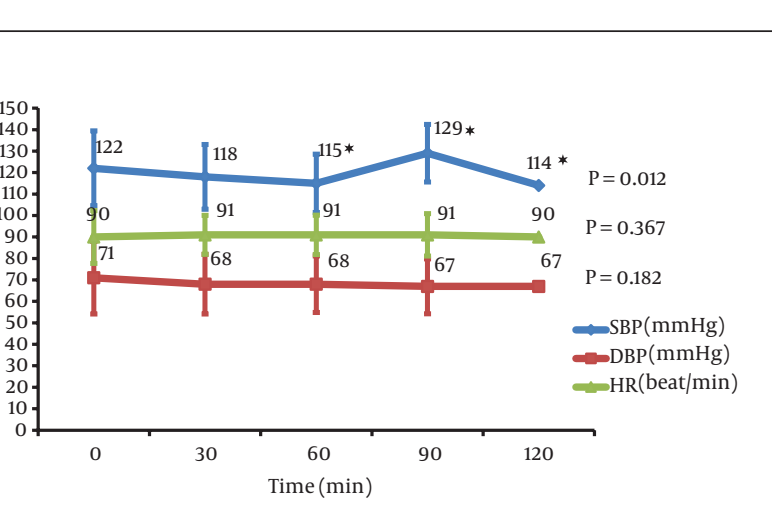

Figure 4. Hemodynamic Parameters Fluctuation in Different Study Time Intervals

\section{Discussion}

Many researches performed with an emphasis on the fact that pain control is not enough in ICU patients, and pain is not assessed adequately by caregivers. A number of researches performed to find out the best way to evaluate pain with physiologic and behavioral criteria in unconscious and tracheal intubated patients.

This study aimed to evaluate the use of two tools, CPOT and FE, and their agreement in detecting and evaluating pain levels in intubated patients in ICU, after cardiac surgery. In this research, intubated patients experienced different levels of pain and when their evaluated pain was in highest and lowest levels, the highest level of agreement observed between the two tools. Whereas, there was lack of strong agreement between the tools when there was moderate and low level pain, so that in T4, the change was reported in the pain by CPOT tool, but FE tool was unable to detect this change. This would suggest higher sensitivity of CPOT tool in assessing pain compared to FE. In the study of Gelinas et al. on intubated patients, over a half of patients experienced pain while resting (18). The researcher here concluded that patients would experience pain during their time in ICU in situations such as low consciousness levels and when trachea was intubated.

Also in Gelinas's study on intubated patients, most nurses expected body movement to detect patient pain and rarely used FE (19). Whereas, Arif-Rahu and Grap specified FE as one of the most widely used methods of pain detection due to its behavioral expression and feelings richness.
However, he considered it as an incomplete tool, since patient's lower half of face (mouth and lips) is covered by tapes used to fix tracheal tube and/or nasogastric tube; therefore, patient's face is not exposed fully to evaluate expressions and muscle movements and recommends FACS (facial action coding system) to evaluate and detect pain using FE, also pain detection is not limited to patient's grimace and patient's face facial muscles retractions (20).

Moreover, as pain is an occurrence that would happen to the whole body and is not limited to one location (presented in chest, lower body parts, hands, and etc.) a pain assessment tool can evaluate whole body reaction, logically. On the other hand, some patients would grimace unaware when they are awake, and every such expression in awareness cannot be interpreted as pain. Thus, all the mentioned reasons and deficiencies for FE tool can cause underestimate and overestimate in results and cause inappropriate administration of analgesic medications for these patients, just like the first step (To) in our study, where FE tool recorded higher level of pain compared to CPOT tool.

Of hemodynamic variables, systolic blood pressure was consistent with pain level changes before and after analgesic drugs were used, and increase in systolic blood pressure was consistent with reported pain increase by CPOT tool. Aurbor and Gelinas showed that physiologic indicators would increase with severe pain (21). Whereas, McCaffrey and Locsin (22) reported that physiologic indicators would be affected by environmental conditions such as physiologic and hemodynamic conditions and medications (analgesics, sedatives and tranquilizers), they are not constant indicators of proving pain and recommend physiologic indicators to detect pain. In this research, the agreement between CPOT and changes in physiologic changes because of pain, like systolic blood pressure, was more than FE, which could suggest that CPOT is more sensitive compared to FE.

Marmo and Fowler evaluated pain in intubated patients after cardiac surgery using three multi-criteria tools, FLACC, NVPS and CPOT, and specified CPOT as a more sensitive tool compared to the other two (8). Boitor et al. established validity of CPOT for evaluation of sensory and affective constituents of pain after cardiac surgery. They found that vital signs were not precise to assess pain and validated tools should be used for this purpose (23). Rijkenberg et al. reported that behavioral pain scale (BPS) and criticalcare pain observation tool (CPOT) are useful pain evalua- 
tion instruments for un-communicative and sedated ICU patients. This study compared validation and consistency of CPOT and BPS in tracheal intubated patients (24).

Pain assessment has always been a challenge in patients hospitalized in ICU, who are unable to communicate adequately and express their pain due to numerous reasons (low level of consciousness, tracheal intubation, etc.). Since pain is a mental and complicated phenomenon usually felt throughout the body, CPOT tool is more sensitive due to having multiple items and evaluating different behavioral indicators for pain in intubated patients compared to FE tool with only one criterion. Moreover, CPOT is more consistent with physiologic changes due to pain in patients. The researcher suggests more studies to confirm the sensitivity of CPOT tool.

There were some limitations in our study, such as research units limited to a single center and only intubated patients undergoing cardiac surgery were studied; therefore, our findings are generalizable to these patients. Second, some behaviors such as stress were considered as pain by the evaluation tool and unpredictable changes in patients' conditions (return to the operating room, administration of tranquilizers instead of analgesics, changing hemodynamics-hypotension or low cardiac output syndrome to make patient fully unawake) happened that resulted in the samples being less than what was originally intended.

\section{Acknowledgments}

With special thanks to Rajaie cardiovascular center intensive care units nurses for their kind cooperation during the study. Special thanks to Dr. Hooman Bakhshandeh for statistical analysis of our data.

\section{Footnote}

Authors' Contribution:Majid Kiavar conducted the study and supervised the project, Rasoul Azarfarin supervised the project and contributed in paper writing and submission of manuscript, Ziae Totonchi was consultant in research project and contributed in data collection, Fatemeh Tavakoli collected the study data and wrote the thesis and manuscript; Azin Alizadehasl contributed in study design and conduction and data collection; Mitra Teymouri contributed in data collection and manuscript writing.

\section{References}

1. Ahlers SJ, van der Veen AM, van Dijk M, Tibboel D, Knibbe CA. The use of the Behavioral Pain Scale to assess pain in conscious sedated patients. Anesth Analg. 2010;110(1):127-33. doi: 10.1213/ ANE.0b013e3181c3119e. [PubMed:19897804]

2. Connor LO. Critical care nurses' judgement of pain status: a case study design. Intensive Crit Care Nurs. 2012;28(4):215-23. doi 10.1016/j.iccn.2012.01.004. [PubMed: 22365765]

3. Aslan FE, Badir A, Arli SK, Cakmakci H. Patients' experience of pain after cardiac surgery. Contemp Nurse. 2009;34(1):48-54 [PubMed: 20230171]

4. Williams TA, Martin S, Leslie G, Thomas L, Leen T, Tamaliunas S, et al. Duration of mechanical ventilation in an adult intensive care unit after introduction of sedation and pain scales. Am J Crit Care 2008;17(4):349-56. [PubMed: 18593834]

5. Herr K, Coyne PJ, Key T, Manworren R, McCaffery M, Merkel S, et al. Pain assessment in the nonverbal patient: position statement with clinical practice recommendations. Pain Manag Nurs. 2006;7(2):4452. doi:10.1016/j.pmn.2006.02.003. [PubMed:16730317]

6. Gelinas C, Tousignant-Laflamme Y, Tanguay A, Bourgault P. Exploring the validity of the bispectral index, the Critical-Care Pain Observation Tool and vital signs for the detection of pain in sedated and mechanically ventilated critically ill adults: a pilot study. Intensive Crit Care Nurs. 2011;27(1):46-52. doi: 10.1016/j. iccn.2010.11.002. [PubMed: 21169020]

7. Young J, Siffleet J, Nikoletti S, Shaw T. Use of a Behavioural Pain Scale to assess pain in ventilated, unconscious and/or sedated patients. Intensive Crit Care Nurs. 2006;22(1):32-9. doi: 10.1016/j. iccn.2005.04.004. [PubMed:16198570]

8. Marmo L, Fowler S. Pain assessment tool in the critically ill post-open heart surgery patient population. Pain Manag Nurs 2010;11(3):134-40. doi: 10.1016/j.pmn.2009.05.007. [PubMed: 20728062]

9. Gelinas C, Harel F, Fillion L, Puntillo KA, Johnston CC. Sensitivity and specificity of the critical-care pain observation tool for the detection of pain in intubated adults after cardiac surgery. J Pain Symptom Manage. 2009;37(1):58-67. doi:10.1016/j.jpainsymman.2007.12.022. [PubMed: 18599262]

10. Pasero C, Coyne PJ. Assessing and Treating the Pain of Pancreatitis. Am J Nurs. 1998;98(11):14. doi: 10.1097/00000446-19981100000007. [PubMed: 9739741]

11. Keane KM. Validity and reliability of the critical care pain observation tool: a replication study. Pain Manag Nurs. 2013;14(4):e21625. doi: 10.1016/j.pmn.2012.01.002. [PubMed: 24315275]

12. Gelinas C, Arbour C, Michaud C, Vaillant F, Desjardins S. Implementation of the critical-care pain observation tool on pain assessment/management nursing practices in an intensive care unit with nonverbal critically ill adults: a before and after study. Int J Nurs Stud. 2011;48(12):1495-504. doi: 10.1016/j. ijnurstu.2011.03.012. [PubMed: 21550048]

13. Rose L, Haslam L, Dale C, Knechtel L, Fraser M, Pinto R, et al. Survey of assessment and management of pain for critically ill adults. Intensive Crit Care Nurs. 2011;27(3):121-8. doi: 10.1016/j. iccn.2011.02.001. [PubMed:21398127]

14. Paulson-Conger M, Leske J, Maidl C, Hanson A, Dziadulewicz L. Comparison of two pain assessment tools in nonverbal critical care patients. Pain Manag Nurs. 2011;12(4):218-24. doi: 10.1016/j. pmn.2010.05.008. [PubMed:22117753]

15. Gelinas C. Nurses' evaluations of the feasibility and the clinical utility of the Critical-Care Pain Observation Tool. Pain Manag Nurs 2010;11(2):115-25. doi:10.1016/j.pmn.2009.05.002. [PubMed: 20510842]

16. Gelinas C, Fillion L, Puntillo K, Vienc C, Fortier M. Validation of the critical care pain observation tool in adults .Am J Crit Care. 4(15):421

17. Prkachin KM. The consistency of facial expressions of pain: a comparison across modalities. Pain. 1992;51(3):297-306. [PubMed: 1491857]

18. Gelinas C, Johnston C. Pain assessment in the critically ill ventilated adult: validation of the Critical-Care Pain Observation Tool and physiologic indicators. Clin J Pain. 2007;23(6):497-505. doi 10.1097|AJP.ob013e31806a23fb. [PubMed: 17575489]

19. Gelinas C, Fortier M, Viens C, Fillion L, Puntillo K. Pain assessment and management in critically ill intubated patients: a retrospective study. Am J Crit Care. 2004;13(2):126-35. [PubMed:15043240]

20. Arif-Rahu M, Grap MJ. Facial expression and pain in the critically ill non-communicative patient: state of science review. Intensive Crit Care Nurs. 2010;26(6):343-52. doi: 10.1016/j.iccn.2010.08.007. [PubMed: 21051234]

21. Arbour C, Gelinas C. Are vital signs valid indicators for the assessment of pain in postoperative cardiac surgery ICU adults? Intensive Crit Care Nurs. 2010;26(2):83-90. doi: 10.1016/j. iccn.2009.11.003. [PubMed:20044256]

22. McCaffrey R, Locsin R. The effect of music on pain and acute confusion in older adults undergoing hip and knee surgery. Holist Nurs Pract. 2006;20(5):218-24. [PubMed: 16974175] 


\section{KiavarM et al.}

23. Boitor M, Fiola JL, Gelinas C. Validation of the Critical-Care Pain Observation Tool and Vital Signs in Relation to the Sensory and Affective Components of Pain During Mediastinal Tube Removal in Postoperative Cardiac Surgery Intensive Care Unit Adults. J Cardiovasc Nurs. 2015. doi: 10.1097/JCN.0000000000000250. [PubMed: 25829139]
24. Rijkenberg S, Stilma W, Endeman H, Bosman RJ, Oudemans-van Straaten HM. Pain measurement in mechanically ventilated critically ill patients: Behavioral Pain Scale versus Critical-Care Pain Observation Tool. J Crit Care. 2015;30(1):167-72. doi: 10.1016/j. jcrc.2014.09.007. [PubMed: 25446372] 